\title{
ANTROPOMETRIA, MATURAÇÃO SEXUAL E IDADE DA MENARCA DE ACORDO COM O NÍVEL SOCIOECONÔMICO DE MENINAS ESCOLARES DE CASCAVEL (PR)
}

Everton Paulo Roman ${ }^{1}$, Roberto Regis Ribeiro², Gil Guerra-Júnior*3 ${ }^{*}$ Antonio de Azevedo Barros-Filho 4

Trabalho elaborado no Departamento de Pediatria da Faculdade de Ciências Médicas (FCM) da Universidade Estadual de Campinas (UNICAMP)

\author{
*Correspondência: \\ Prof. Dr. Gil Guerra-Junior \\ Departamento de \\ Pediatria - FCM \\ Universidade Estadual de \\ Campinas (UNICAMP) - \\ CP 6111 \\ Cidade Universitária \\ "Zeferino Vaz" \\ Campinas - São Paulo \\ CEP 13083-970 \\ Telefone (19)3521-8923 \\ Fax (19)3521-8925 \\ gilguer@fcm.unicamp.br
}

\begin{abstract}
RESUMO
OвJetivo. Avaliar a idade da menarca e a antropometria de meninas escolares de diferentes níveis socioeconômicos em Cascavel (Estado do Paraná) - Região Sul do Brasil.

MÉtodos. Estudo transversal realizado no ano de 2006 com 2.761 meninas em 27 escolas públicas e particulares. A idade da menarca foi obtida pelo método status quo. Para a avaliação da maturação sexual foi realizada a autoavaliação de acordo com os critérios descritos por Tanner. O nível socioeconômico foi obtido por meio do questionário da ABEP (2003). Avaliou-se o peso e a estatura, obtendo-se $\mathrm{o}$ índice de massa corporal (IMC). Foram utilizados a análise estatística descritiva e o teste de Mann-Whitney, com nível de significância de 5\%.

Resultados. A idade da menarca geral foi $12,2 \pm 1,2$ anos e $12,1 \pm 1,1 ; 12,3 \pm 1,2$ e $12,4 \pm 1,2$ anos para os tercis alto, médio e baixo de nível socioeconômico, respectivamente. Foram constatados valores superiores para as meninas pós-menarca no peso e na estatura dos 10 aos 17 anos de idade e no IMC dos 11 aos 14 anos de idade.

ConcLusões. As meninas de nível socioeconômico alto apresentaram idade da menarca em idade inferior aos demais níveis socioeconômicos. Embora estas diferenças tenham sido observadas em níveis socioeconômicos distintos, biologicamente foram consideradas pequenas.
\end{abstract}

UnItermos: Menarca. Puberdade. Índice de massa corporal. Estatura. Adolescente.

\section{INTRODUÇÃO}

A puberdade é um evento fisiológico do processo de maturação sexual que começa com a ativação do eixo hipotálamohipófise-gônada e culmina com a capacidade reprodutiva. Durante a puberdade as mudanças são dinâmicas e podem variar quanto à idade de início ou término, à magnitude, à velocidade que se expressam e suas inter-relações, além da etnia, do gênero e do meio ambiente em que o indivíduo vive. A puberdade abrange o desenvolvimento das gônadas, dos órgãos sexuais e das características sexuais secundárias, sendo estas, nas mulheres, caracterizadas pelo desenvolvimento das mamas (telarca), dos pêlos pubianos (pubarca) e a ocorrência da primeira menstruação (menarca). Dentre esses eventos, a menarca é o fenômeno mais representativo e de mais fácil determinação, constituindo um importante indicador da maturação sexual, especialmente em estudos históricos e/ou retrospectivos ${ }^{1,2}$.

A idade do surgimento da menarca diminuiu tanto nos países desenvolvidos quanto nos países em desenvolvimento, assim como no Brasil ${ }^{3}$. Esta antecipação da idade da menarca foi mais expressiva entre o final do século XIX e os anos 70 do século $X X^{4-6}$. A partir desta época, não há consenso entre os estudos se a idade da primeira menstruação continua a diminuir, mesmo que mais lentamente, ou se está estacionada ${ }^{2,4-7}$.

Além de ser um indicador de maturação biológica, a idade da menarca também é usada para mostrar as mudanças socioeconômicas que ocorrem com as populações. Existem poucos estudos que comparam a idade da menarca segundo o nível socioeconômico em um mesmo país e mesmo assim estes estudos apresentam resultados controversos ${ }^{8-10}$. Alguns estudos tentam associar a antecipação da menarca à melhora do nível socioeconômico e a maior prevalência de obesidade ${ }^{10-14}$.

A cidade de Cascavel está localizada na região oeste do Estado do Paraná, Região Sul do Brasil e de acordo com o Instituto Brasileiro de Geografia e Estatística ${ }^{15}$ tem população estimada em aproximadamente 285 mil habitantes. Apresenta colonização recente, principalmente por migrantes descendentes de italianos provenientes do Estado do Rio Grande do Sul, a partir de 1950.

1. Doutor em Saúde da Criança e do Adolescente - Faculdade de Ciências Médicas (FCM) - Universidade Estadual de Campinas (UNICAMP), São Paulo, SP

2. Aluno de Doutorado em Saúde da Criança e do Adolescente - Faculdade de Ciências Médicas (FCM) - Universidade Estadual de Campinas (UNICAMP), São Paulo, SP

3. Professor Associado do Departamento de Pediatria - Faculdade de Ciências Médicas (FCM) - Universidade Estadual de Campinas (UNICAMP), São Paulo, SP

4. Professor Associado do Departamento de Pediatria - Faculdade de Ciências Médicas (FCM) - Universidade Estadual de Campinas (UNICAMP), São Paulo, SP 
Atualmente, a cidade é considerada pólo regional de indústrias no agronegócio, comércio e prestação de serviços.

Pela necessidade de conhecer melhor a associação que o nível socioeconômico exerce nas variáveis de peso, estatura e índice de massa corporal (IMC) em relação à idade da menarca, o objetivo deste estudo foi avaliar a idade da menarca e a antropometria em meninas escolares de nove a 17 anos de idade de diferentes níveis socioeconômicos da cidade de Cascavel (PR).

\section{MÉTOdos}

Trata-se de um estudo transversal realizado no ano de 2006 em 27 escolas (13 públicas e 14 particulares) na cidade de Cascavel, Oeste do Estado do Paraná - Região Sul do Brasil. Este estudo foi realizado com meninas que frequentavam da $3^{\mathrm{a}}$ série do ensino fundamental até o $3^{\circ}$ ano do ensino médio.

Para a sua realização foram considerados como elementos pertencentes da população todas as escolares de nove a 17 anos de idade do sexo feminino que estivessem matriculadas nas instituições de ensino que participaram da pesquisa. 0 agrupamento das idades foi estabelecido pelos critérios estabelecidos por Ross e Marfell-Jones ${ }^{16}$ em cada idade utilizando os intervalos de $-0,50$ a 0,49.

De acordo com o setor de Estatística do Núcleo Regional de Ensino da Secretaria de Educação do Estado do Paraná da cidade de Cascavel, a população deste estudo constituía-se de aproximadamente 16.000 meninas da rede pública e de 4.500 na rede particular ${ }^{15}$. Para o cálculo da amostra foram utilizados os procedimentos descritos por Barbetta ${ }^{17}$. As escolas foram selecionadas por sorteio. Foram entregues 3.250 termos de consentimento livre e esclarecido e 2.849 escolares apresentaram o documento assinado pelos pais/responsáveis autorizando a participação no estudo, sendo que destas, 88 foram excluídas da análise final dos dados, pois apresentavam idade inferior a nove ou superior a 17 anos. A amostra final foi constituída de 2.761 escolares.

A coleta foi realizada no horário de aula, no período da manhã, quando as meninas foram conduzidas até uma sala designada para realizar a avaliação. Foram avaliados o peso e a estatura e, com estes dados, foi calculado o índice de massa corporal $\left[I M C=\right.$ peso $(\mathrm{kg}) /$ estatura $\left.(\mathrm{m})^{2}\right]$. A idade média da menarca foi obtida pelo método "status quo" que questionava a presença ou não de menstruação. Para as respostas positivas utilizou-se um questionário retrospectivo procurando identificar o dia, o mês e o ano de sua ocorrência. O estadio de maturação sexual foi obtido por autoavaliação com a comparação das fotos dos cinco estadios de desenvolvimento das mamas (M1 a M5), de acordo com os critérios descritos por Tanner ${ }^{18}$.

A avaliação do nível socioeconômico foi realizada com um questionário respondido pelos pais/responsáveis, utilizando-se o Critério de Classificação Econômica do Brasil proposto pela Associação Brasileira de Empresas de Pesquisa ${ }^{19}$ que avalia o nível de escolaridade do chefe da família, o número de empregadas mensalistas e nove itens referentes a bens materiais, sendo a pontuação obtida dividida nos tercis considerados como alto, médio e baixo.

Os dados foram armazenados no programa SPSS for Windows versão 13.0. Foi realizada a análise descritiva dos dados com cálculos de média, desviopadrão, percentual e construção de tabelas de frequência. O teste não-paramétrico de
Mann-Whitney foi empregado para avaliar a diferença da idade da menarca entre os níveis socioeconômicos, e para avaliar o peso, a estatura e o IMC entre as meninas com e sem menarca nas diferentes idades. 0 nível de significância adotado para 0 estudo foi $p<0,05$.

Todas as meninas envolvidas na pesquisa e seus responsáveis foram informados quanto aos objetivos do estudo e esclarecidos quanto aos métodos utilizados, ficando garantido às participantes o direito de desistir do estudo a qualquer momento. Este estudo foi aprovado pelo Comitê de Ética em Pesquisa da FCM-UNICAMP sob o parecer 568/2003.

\section{Resultados}

Na Tabela 1 observa-se o aumento gradual dos nove aos 17 anos de idade de meninas com a presença de menarca, sendo que aos 13 anos de idade aproximadamente $63 \%$ das meninas já tinham apresentado a menarca, e aos 14 anos estes valores ficaram próximos a 90\%, independente do nível socioeconômico.

Quando comparados os valores das médias da idade da menarca entre os níveis socioeconômicos, observou-se diferença superior e estatisticamente significativa entre as meninas de nível socioeconômico alto $(12,1 \pm 1,1)$ em relação às que pertenciam ao nível socioeconômico baixo $(12,4 \pm 1,2) \quad(p=0,004)$. No entanto, esta diferença não foi identificada entre o nível socioeconômico médio $(12,3 \pm 1,2)$ e o alto $(p=0,164)$ e entre o médio e o baixo $(p=0,443)$. A idade média da menarca entre as meninas, não considerando o agrupamento dos níveis socioeconômicos, foi de 12,2 $\pm 1,2$ anos.

Foram observados valores superiores e estatisticamente significativos para as meninas pós-menarca nas variáveis de peso e estatura dos 10 aos 17 anos de idade e no IMC dos 11 aos 14 anos de idade (Tabela 2).

Quando se analisou a presença da menarca associada à maturação sexual (estadios das mamas) observou-se que 63,7\% das meninas que afirmaram já ter apresentado a menarca encontravam-se no estadio M4 (mediana de 2,0 anos com valor mínimo de 0,01 e máximo de 7,6 anos em relação à ocorrência da menarca). Por outro lado, notou-se também que aquelas pertencentes aos estadios M1 a M3 e que já tiveram a menarca corresponderam a $14,1 \%$ (Tabela 3).

\section{Dıscussão}

Neste estudo, procurou-se verificar a relação da idade da menarca e dos dados antropométricos em relação aos distintos níveis socioeconômicos, pois se sabe que o ambiente exerce grande influência nos resultados relativos à maturação sexual e à composição corporal. As meninas que participaram desta pesquisa pertenciam à área urbana da cidade de Cascavel (PR). Como o Brasil é um país de contrastes, e sendo a cidade escoIhida localizada em uma das regiões mais ricas do país, para alcançar os objetivos do estudo, as avaliações foram realizadas em escolas que apresentavam realidades diferentes em relação ao nível socioeconômico.

Os resultados encontrados neste estudo apontaram que a média geral de ocorrência da idade da menarca foi de 12,2 $\pm 1,2$ anos e de $12,1 \pm 1,1,12,3 \pm 1,2 ; 12,4 \pm 1,2$ anos para os níveis socioeconômicos alto, médio e baixo, respectivamente. Em 


\begin{tabular}{|c|c|c|c|c|c|c|c|c|c|}
\hline \multirow[b]{4}{*}{ idade } & \multicolumn{8}{|c|}{ nível socioeconômico } & \\
\hline & \multicolumn{3}{|c|}{$\begin{array}{c}\text { Alto } \\
\mathrm{n}=948\end{array}$} & \multicolumn{3}{|c|}{$\begin{array}{c}\text { Médio } \\
\mathrm{n}=757\end{array}$} & \multicolumn{3}{|c|}{$\begin{array}{c}\text { Baixo } \\
\mathrm{n}=1.056\end{array}$} \\
\hline & \multicolumn{3}{|c|}{ menarca } & \multicolumn{3}{|c|}{ menarca } & \multicolumn{3}{|c|}{ menarca } \\
\hline & $\mathrm{n}$ & $n$ & (\%) & $\mathrm{n}$ & $\mathrm{n}$ & (\%) & $\mathrm{n}$ & $\mathrm{N}$ & (\%) \\
\hline 9 & 75 & 0 & 0 & 58 & 0 & 0 & 99 & 1 & 0,9 \\
\hline 10 & 123 & 23 & 18,7 & 67 & 2 & 2,9 & 130 & 1 & 0,7 \\
\hline 11 & 98 & 14 & 14,3 & 97 & 11 & 11,3 & 168 & 13 & 7,7 \\
\hline 12 & 108 & 47 & 43,5 & 98 & 28 & 28,6 & 171 & 44 & 25,7 \\
\hline 13 & 133 & 83 & 62,4 & 105 & 69 & 65,7 & 139 & 87 & 62,9 \\
\hline 14 & 118 & 105 & 88,9 & 86 & 80 & 93,0 & 113 & 103 & 91,1 \\
\hline 15 & 133 & 128 & 96,2 & 104 & 99 & 95,2 & 102 & 100 & 98,0 \\
\hline 16 & 98 & 93 & 94,9 & 93 & 92 & 98,9 & 73 & 72 & 98,6 \\
\hline 17 & 62 & 59 & 95,1 & 49 & 49 & 100,0 & 61 & 61 & 100 \\
\hline
\end{tabular}

Tabela 2 - Valores de média e desvio-padrão de estatura, peso e IMC de meninas entre 9 e 17 anos de idade pré e pós-menarca

\begin{tabular}{|c|c|c|c|c|c|c|}
\hline \multirow[b]{2}{*}{ idade } & \multicolumn{2}{|c|}{ Estatura } & \multicolumn{2}{|c|}{ Peso } & \multicolumn{2}{|c|}{ IMC } \\
\hline & pré & pós & Pré & pós & pré & pós \\
\hline 9 & $135,4 \pm 6,4$ & $136,8 \pm 1,2$ & $31,0 \pm 6,2$ & $32,5 \pm 1,2$ & $16,7 \pm 2,5$ & $17,3 \pm 1,2$ \\
\hline 10 & $140,1 \pm 7,4$ & $151,3 \pm 5,5^{*}$ & $33,4 \pm 7,1$ & $49,2 \pm 10,9 *$ & $16,8 \pm 2,5$ & $18,1 \pm 3,0$ \\
\hline 11 & $145,4 \pm 7,3$ & $156,6 \pm 6,9 *$ & $36,6 \pm 7,6$ & $50,9 \pm 11,2^{*}$ & $17,2 \pm 2,8$ & $19,9 \pm 3,0 *$ \\
\hline 12 & $150,5 \pm 6,9$ & $156,9 \pm 6,1^{*}$ & $40,4 \pm 8,4$ & $49,9 \pm 9,7 *$ & $17,8 \pm 3,0$ & $20,1 \pm 3,5^{*}$ \\
\hline 13 & $154,4 \pm 8,1$ & $159,8 \pm 5,9 *$ & $42,0 \pm 8,0$ & $50,9 \pm 9,5^{*}$ & $17,7 \pm 2,8$ & $19,8 \pm 3,3^{*}$ \\
\hline 14 & $153,7 \pm 7,9$ & $160,9 \pm 5,6^{*}$ & $41,7 \pm 8,2$ & $51,9 \pm 8,2^{*}$ & $18,1 \pm 3,4$ & $20,0 \pm 3,1$ * \\
\hline 15 & $153,6 \pm 11,9$ & $161,9 \pm 6,0 *$ & $41,1 \pm 7,2$ & $53,8 \pm 8,7^{*}$ & $18,3 \pm 3,2$ & $20,5 \pm 3,0$ \\
\hline 16 & $155,5 \pm 9,9$ & $163,1 \pm 5,7^{*}$ & $42,6 \pm 8,4$ & $54,9 \pm 8,7 *$ & $20,1 \pm 1,9$ & $20,6 \pm 3,2$ \\
\hline 17 & $144,0 \pm 4,5$ & $162,5 \pm 5,6^{*}$ & $34,2 \pm 4,9$ & $54,6 \pm 8,1^{*}$ & $21,4 \pm 0,8$ & $20,5 \pm 2,6$ \\
\hline
\end{tabular}

*diferenças estatísticas na mesma idade entre pré e pós-menarca $(p<0,05)$

Tabela 3 - Valores de mediana, mínimo e máximo do tempo (em anos) de ocorrência da menarca e a frequência absoluta e relativa da presença ou não da menarca de acordo com os estadios de desenvolvimento das mamas

\begin{tabular}{|c|c|c|c|c|c|c|c|c|c|c|}
\hline & & \multirow{2}{*}{\multicolumn{3}{|c|}{ Tempo de ocorrência da menarca (anos) }} & \multicolumn{6}{|c|}{ Menarca } \\
\hline & & & & & \multicolumn{2}{|c|}{$\operatorname{sim}$} & \multicolumn{2}{|c|}{ não } & \multicolumn{2}{|c|}{ total } \\
\hline \multirow{4}{*}{ Estadio de mamas } & M1 & 0,7 & 0,5 & 1,3 & 4 & 0,3 & 422 & 32,5 & 426 & 15,4 \\
\hline & M3 & 0,6 & $<0,1$ & 5,7 & 184 & 12,6 & 305 & 23,5 & 489 & 17,7 \\
\hline & M4 & 2,0 & $<0,1$ & 7,6 & 932 & 63,7 & 68 & 5,2 & 1.000 & 36,3 \\
\hline & M5 & 3,1 & $<0,4$ & 7,4 & 327 & 22,3 & 1 & 0,1 & 328 & 11,8 \\
\hline
\end{tabular}

2005, Oliveira e Veiga ${ }^{20}$, na cidade do Rio de Janeiro, mostraram que meninas que estudavam em escolas particulares $(11,5 \pm 1,2$ anos) tendiam a apresentar a menarca antes das meninas de

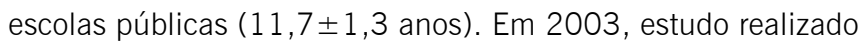
na cidade de Marechal Cândido Rondon (PR) observou idade média da menarca de 12,2 $\pm 0,8$ anos $^{21}$. Em Londrina, cidade localizada no norte do Paraná, estudo realizado por Borges e Pires Júnior $^{22}$ no ano 2000 detectou idade da menarca de $12,1 \pm, 1$ anos em meninas de alto nível socioeconômico. Em 1999, Petroski et al. ${ }^{23}$ mostraram que as meninas de escolas públicas da grande Florianópolis (SC) apresentaram idade da menarca de $12,6 \pm 1,1$ anos.

Há de se destacar que existem poucos estudos populacionais que se referem à maturação sexual de meninas brasileiras. As diferenças nos métodos e no tamanho da amostra destes estudos dificultam a melhor interpretação da tendência de antecipação da idade da menarca nas mais diferentes regiões do Brasil ${ }^{20-29}$. Apesar da amostra reduzida em alguns estudos, pode-se observar uma tendência gradativa à diminuição dos valores médios da idade da menarca nos últimos 25 anos, de 13 anos em $1983^{24}$ para $11,7^{20}$ 
em 2005 e 12,2 no presente estudo. No Brasil, o presente estudo compreendeu o maior número de meninas já avaliadas em um único estudo e foi o primeiro a comparar os dados de idade da menarca aos diferentes níveis socioeconômicos.

Pode-se considerar que a região oeste do Paraná está entre as regiões mais ricas e desenvolvidas do Brasil. Quando foi comparada a média da idade da menarca das meninas deste estudo esta foi menor do que a média constatada em estudos realizados em outros países considerados em desenvolvimento. Em 2004, Chavarro et al. ${ }^{30}$ encontraram idade da menarca de $12,7 \pm 1,3$ anos em universitárias colombianas. Em países considerados menos desenvolvidos, Chowdhury et al. ${ }^{31}$ no ano 2000, observaram idade da menarca de 13,0 $\pm 0,9$ anos em meninas da área rural de Bangladesh. Em países com diferentes realidades culturais, Ersoy et al. ${ }^{9}$, em 2004, apontaram a idade da menarca de $12,9 \pm 1,1$ anos em meninas turcas, enquanto que Ayatollahi et al. ${ }^{32}$, no ano de 2002 , encontraram idade média

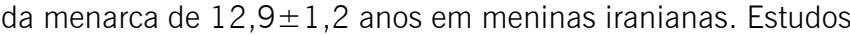
publicados em 2008 mostraram as seguintes idades médias da menarca: na Grécia ${ }^{33}, 12,3 \pm 1,2$ anos; no Irã ${ }^{34}, 12,5 \pm 1,1$ anos e na Tailândia, $13,0 \pm 1,3$ anos.

Em relação à faixa etária de ocorrência da idade da menarca, a literatura aponta que 95\% das meninas tendem a apresentar a menarca entre os 11 e 15 anos de idade cronológica². As informações obtidas neste estudo estão de acordo com a literatura, ou seja, observou-se que aos 15 anos de idade, 95,6\% das meninas já haviam apresentado a menarca, e, quando divididas pelo nível socioeconômico, 96,2\% no alto, 95,2\% no médio e 98\% no baixo, mostrando que apesar da média da idade da menarca ser mais baixa no nível alto, a maior parte das meninas de todos os níveis, mas em especial do nível baixo, tendem a apresentar menarca antes dos 15 anos.

Além de ser um indicador de maturação biológica, a idade da menarca também é usada para mostrar as mudanças socioeconômicas que ocorrem com as populações. Existem poucos estudos que comparam a idade da menarca segundo o nível socioeconômico em um mesmo país, e mesmo assim estes estudos apresentam resultados controversos ${ }^{8-10}$. Alguns estudos tentam associar a antecipação da menarca com a melhora do nível socioeconômico e a maior prevalência de obesidade ${ }^{10-14}$.

Apesar da literatura mostrar que a maturação sexual tende a ocorrer mais precocemente quanto melhor for considerado o nível socioeconômico no qual o indivíduo está inserido, outros estudos mostram resultados diferentes ${ }^{8-14}$. No Brasil, estudos demonstraram tendência à antecipação da menarca mesmo em níveis socioeconômicos menos privilegiados ${ }^{35,36}$. Embora os resultados apresentados no presente estudo abordem que a maturação sexual tende a ocorrer antecipadamente em meninas que pertencem ao nível socioeconômico alto, observa-se que a diferença entre os níveis médio e baixo foi relativamente pequena.

Quando comparados os resultados de peso, estatura e IMC das meninas pré-menarca em relação às pós-menarca, observaram-se, na mesma faixa etária, valores maiores de todos os índices antropométricos nas pós-menarca. Resultados semeIhantes foram observados por Chowdhury et al. ${ }^{31}$ na zona rural de Bangladesh e por Hesketh et al. ${ }^{37}$ na China. Estudo brasileiro de Junqueira do Lago et al. ${ }^{38}$, em 2007, mostra associação entre a idade da menarca e a estatura definitiva.
Quanto à presença da menarca associada aos estadios de desenvolvimento das mamas, 63,7\% das meninas que tiveram menarca relataram pertencer ao estadio 4. Estes resultados são próximos aos valores encontrados na literatura. Em 1984, Colli39 , em um estudo com 3.368 meninas com avaliação da maturação sexual realizada por pediatras, observou que $77,1 \%$ das meninas que haviam tido a menarca estavam no estadio 4 de mamas. Como era de se esperar, apenas 14,1\% apresentaram menarca nos estadios 1 a 3, sendo apenas 1,5\% nos estadios 1 e 2. Vitalle et al. ${ }^{26}$ encontraram $24,7 \%$ das meninas com menarca nos estadios 1 a 3 de mamas.

Neste estudo, alguns fatores que poderiam interferir nos resultados não foram avaliados e merecem ser considerados em análises futuras, como a ordem de nascimento das meninas avaliadas, idade da menarca de suas mães e a cor da pele.

\section{Conclusão}

De acordo com os resultados encontrados neste estudo, as meninas escolares de nível socioeconômico alto tiveram, em média, a menarca antes das meninas que pertenciam aos níveis socioeconômicos médio e baixo. No que se refere às variáveis de antropometria, as meninas que já tiveram a menarca, quando comparadas àquelas que não tiveram, apresentaram valores superiores de peso, estatura e no IMC na maioria das idades, mas principalmente após os 10 anos de idade. A maioria das meninas com menarca estavam no estádio 4 de mamas. No entanto, mesmo apresentando algumas diferenças no ponto de vista estatístico, as diferenças biológicas entre os níveis socioeconômicos podem ser consideradas pequenas.

A melhora nas condições socioeconômicas e a estabilização da economia alcançada pelo país, principalmente nos últimos anos pode ter contribuído, possivelmente, para tais resultados, além do que, esta é uma tendência na maioria dos países desenvolvidos e em países que estão em processo acelerado de desenvolvimento, como é o caso do Brasil.

\section{Agradecimentos}

Os autores agradecem aos diretores, professores e demais funcionários das escolas públicas e particulares da cidade de Cascavel (PR), ao grupo que auxiliou na coleta dos dados e aos pais/responsáveis que autorizaram a participação das meninas para a realização deste estudo.

\section{Conflito de interesse: não há}

\section{SUMmary}

ANTROPOMETRY, SEXUAL MATURATION AND MENARCHEAL AGE ACCORDiNG to SOCIOECONomic Status of SCHOOLGIRLS FRom CascaVel (PR)

OBJECTIVE. To evaluate age of menarche and anthropometric data of schoolgirls of different socioeconomic levels in Cascavel, state of Parana, in Southern Brazil.

Methods. A cross-sectional study was carried out in 2006 with 2,761 girls evaluated in 27 private and public schools. Age at menarche was obtained by the status quo method. Sexual maturity was assessed by self evaluation, according to Tanner's criteria. Socioeconomic level was obtained through the ABEP (2003) questionnaire. Data of weight, stature and Body Mass 
Index (BMI) were obtained. Data were analyzed by descriptive statistics and Mann-Whitney test at 5\% significance.

RESULTS. Age at menarche was $12.2 \pm 1.2$ years in the whole sample, and was $12.1 \pm 1.1 ; 12.3 \pm 1.2$ and $12.4 \pm 1.2$ in girls from high, medium and low socioeconomic levels, respectively. Post-menarche girls aged 10-17 years had higher values of stature and weight and girls aged 11-14 years had higher BMI.

Conclusions. Age at menarche was lesser in girls from a higher socioeconomic level. Although these differences had been found in distinct socioeconomic levels, they were considered biologically small. [Rev Assoc Med Bras 2009; 55(3): 317-21]

KEY WORDS: Menarche. Puberty. Body mass index. Body height. Adolescent.

\section{REFERÊNCIAS}

1. Ellis BJ. Timing of pubertal maturation in girls: an integrated life history approach. Psychol Bull. 2004;130:920-58.

2. Parent AS, Teilmann G, Juul A, Skakkebaek NE, Toparri J, Bourguignon JP. The timing of normal puberty and the age limits of sexual precocity: variations around the world, secular trends, and changes after migration. Endocr Ver. 2003;24:668-93.

3. Carvalho WRG, Farias ES, Guerra-Júnior G. A idade da menarca está diminuindo? Rev Paul Pediatr. 2007;25:76-81.

4. Gluckman PD, Hanson MA. Evolution, development and timing of puberty. Trends Endocrinol Metab. 2006;17:7-12.

5. De Munich Keizer SM, Mul D. Trends in pubertal development in Europe. Hum Reprod Update. 2001;7:287-91.

6. Kalberg J. Secular trends in pubertal development. Horm Res. 2002;57:19-30.

7. Anderson SE, Must A. Interpreting the continued decline in the average age at menarche: results from two nationally representative surveys of U.S. girls studied 10 years apart. J Pediatr. 2005;147:753-60.

8. Wronka I, Pawlinska-Chmara R. Menarcheal age and socio-economic factors in Poland. Ann Hum Biol. 2005;32:630-8.

9. Ersoy B, Balkan C, Guay T, Onag A Egemen A. Effects of different socioeconomic conditions on menarche in Turkish female students. Early Hum Dev. 2004;76:115-25

10. Hernandéz MI, Unanue N, Gaete X, Cassorla F, Codner E. Edad de la menarquia y su relación com el nível socioeconômico e índice de masa corporal. Rev Med Chile. 2007;135:1429-36.

11. Kirchengast S, Bauer M. Menarcheal age is associated with body composition parameters but not with socioeconomic status. Coll Antropol. 2007;31:419-25.

12. Pierce MB, Leon DA. Age at menarche and adult BMI in the Aberdeen children of the 1950s cohort study. Am J Clin Nutr. 2005;82:733-9.

13. Okasha M, McCarron P, McEwen J, Smith GD. Age at menarche: secular trends and association with adult anthropometric measures. Ann Hum Biol. $2001 ; 28: 68-78$

14. Laitinen J, Power C, Jarvelin MR. Family social class, maternal body mass index, childhood body mass index and age at menarche as predictors of adult obesity. Am J Clin Nutr. 2001;74:287-94.

15. Instituto Brasileiro de Geografia e Estatística (IBGE). Censo 2000: estimativa para 2007. Cascavel. [citado 1 ago 2008]. Disponível em:http:www.ibge. gov.br.

16. Ross WD, Marfell-Jones MJ. Kinanthropometry. In: McDougall JD, Wenger HA, Green $\mathrm{HJ}$, editors. Physiological testing of the elite athlete. Ottawa: Mutual; 1982.p.75-115.

17. Barbetta PA. Estatísticas aplicadas às ciências sociais. $5^{a}$ ed. Florianópolis: UFSC; 2003.
18. Tanner JM. Growth at adolescence. $2^{\text {nd }}$ ed. Oxford: Blackwell Scientific Publications; 1962.

19. Associação Brasileira de Pesquisas Econômicas (ABEP). Critério de classificação econômica Brasil. [citado 10 jul 2007]. Disponível em http://www. abep.org.

20. Oliveira CS, Veiga GV. Estudo nutricional e maturação sexual de adolescentes de uma escola pública e de uma escola privada do município do Rio de Janeiro. Rev Nutr. 2005;18:183-91.

21. Borges GA, Schwarztbach C. Idade da menarca em adolescentes de Marechal Cândido Rondon - Pr. Rev Bras Cineantr Des Hum. 2003;5:15-21.

22. Borges GA, Pires Junior R. Idade da menarca em adolescentes de Londrina. Rev Bras Ativ Fis Saude. 2000;5:5-11.

23. Petroski EL, Velho NM, De Bem MFL. Idade de menarca e satisfação com o peso corporal. Rev Bras Cineantr Des Hum. 1999;1:30-6.

24. Scaf G, Freitas JAS, Damante, JH. Determinação da idade da menarca em meninas brancas, brasileiras, da região de Bauru. Pediatria (São Paulo). 1983:5:169-74.

25. Souza EAC, Medeiros Filho JG, Souza ESM. Estudo da maturação sexual em escolares do sexo feminino no município de João Pessoa - Paraíba. Pediatria (São Paulo). 1996;18:59-64.

26. Vitalle MSS, Tomioka CY, Juliano Y, Amancio OMS. Índice de massa corporal, desenvolvimento puberal e sua relação com a menarca. Rev Assoc Med Bras. 2003; 49: 429-33

27. Biassio LG, Matsudo SMM, Matsudo VKR. Impacto da menarca nas variáveis antropométricas e neuromotoras da aptidão física, analisado longitudinalmente. Rev Bras Ciencia Mov. 2004;12:97-101.

28. Moreira DM, Fragoso MIJ, Oliveira Júnior AV. Níveis maturacional e socioeconômico de jovens sambistas do Rio de Janeiro. Rev Bras Med Esporte. 2004;10:16-23.

29. Castilho SD, Saito MI, Barros Filho AA. Crescimento pós-menarca em uma coorte de meninas Brasileiras. Arq Bras Endocrinol Metab 2005:49:971-7.

30. Chavarro J, Villamor E, Narváez J, Hoyos A. Socio-demographic predictors of age at menarche in a group of Colombian university women. Ann Hum Biol. 2004; 31:245-57.

31. Chowdhury S, Shahabuddin AKM, Seal AJ, Talukder KK, Hassan Q, Begum RA, et al. Nutritional status and age at menarche in rural area of Bangladesh. Ann Hum Biol. 2000;27:249-56.

32. Ayatollahi SMT, Dowlatabadi E, Ayatollahi SAR. Age at menarche in Iran. Ann Hum Biol. 2002;29:355-62.

33. Papadimitriou A, Fytanidis G, Douros K, Bakoula C, Nicolaidou P, Fretzayas A. Age at menarche in contemporary Greek girls: evidence for leveling-off the secular trend. Acta Paediatr. 2008;97:812-5.

34. Delevar MA, Hajian-Tilaki KO. Age at menarche in girls from 1985 to 1989 in Mazandaran, Islamic Republic of Iran. East Mediterr Health J. 2008;14:90-4.

35. Junqueira do Lago M, Faerstein E, Souza Lopes C, Werneck GL. Family socio-economic background modified secular trends in age at menarche: evidence from the Pró-Saúde Study (Rio de Janeiro, Brazil). Ann Hum Biol. 2003;30:347-52.

36. Kac G, Coelho ASC, Velasques-Melendes G. Secular trend in age at menarche for women born between 1920 and 1979 in Rio de Janeiro, Brazil. Ann Hum Biol. 2000;27:423-28.

37. Hesketh T, Ding QJ, Tomkins A. Growth status and menarche in urban and rural China. Ann Hum Biol. 2002;29:348-52.

38. Junqueira do Lago, M, Faerstein E, Sichieri R, Lopes CS, Werneck G. Associação entre idade da menarca e estatura definitiva no estudo Pró-Saúde. Rev Assoc Med Bras. 2007;53:20-4.

39. Colli AS. Inter-relações entre características de maturação sexual em adolescentes brasileiros. II- Sexo feminino. Pediatria (São Paulo). 1984;6:63-8

Artigo recebido: 19/04/08 Aceito para publicação: 19/11/08 